\title{
A Novel Protocol for Safety Messaging and Secure Communication for VANET System : DSRC
}

\author{
Venkatamangarao Nampally \\ Research Scholar, Department of Computer Science \\ University College of Science, Osmania University \\ Hyderabad, India.
}

\author{
Dr. M. Raghavender Sharma \\ Asst. Professor, Department of statistics \\ University College of Science, Osmania University \\ Hyderabad, India.
}

\begin{abstract}
DSRC is a one-to-many way powerful, efficient, and robust novel protocol using wireless technology which provides high data transmission capability from source node to target node in VANET system environment. In VANET, it is intended as highly data secure protocol in communication, highspeed wireless communication technology among users and infrastructure of VANET. And it is considered by many researchers and scientists as a traditional protocol for VANETs and the most viable approach to low latency short range vehicular networks. One approach to increase the safety is alerting the drivers in emergency conditions before they attain the destination. In this paper, we discuss DSRC technology in possible dimensions.
\end{abstract}

Keywords- DSRC; ITS; VANET; CEN 278; Wireless Technology; ETC; Viable; OFDM.

\section{INTRODUCTION}

VANET system is a new wireless technology which connects the vehicular nodes on the basis of short-range wireless communication (IEEE 802.11p). Now-a-days our country is tremendously developing in providing better services to roadside users. The vehicular ad hoc network is ad hoc, local, highly mobile with large number of contending nodes. In VANET environment, it is possible strategically place appropriate infrastructure at roadside and provide better services to its users by using these infrastructure. DSRC is a novel protocol for sending and receiving safety messages in VANET communication criteria [1].

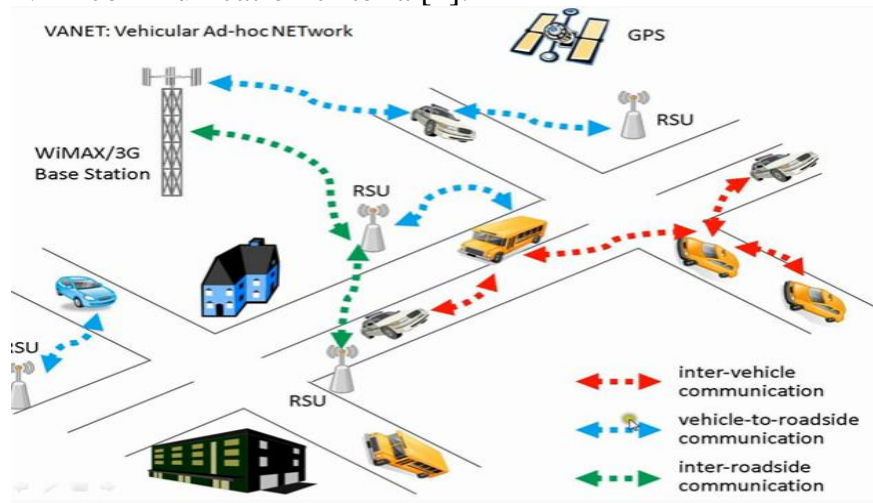

Fig. 1. Communication scenario in VANET

DSRC allows vehicles properly communicate with each other with or without using infrastructure. It offers the efficient, robust, and potentially effective benefits in Vehicular communication Technology which increases overall safety on road and reliable efficiency of the transportation system in VANET system communication. And also it provides excellent services in all type communications of VANET; especially it gives excellent support for both V2V and V2R communications [2]. In both communication scenarios, a BSM (Basic Safety Message) will be sent in 360 degrees pattern using IEEE 802.11p technology. Actually DSRC has developed to eliminate the problems of safety, environmental, and mobility problems of VANET system. Like DSRC technology, ITS also provide and control the traffic conflicts, collecting amount at toll gates in an electronic way, and crash notifications in emergency conditions along with infotainment services. The goal of ITS [3] is to incorporate the technology into the transportation infrastructure of VANET system to improve its safety conditions. Sensors are used to detect traffic jams and emergency conditions. The limited number of vehicles with transceivers led to fragmentation of a total network or some portion of network. All protocols used in VANET system may not assume that vehicles should communicate with each other in a proper way. So, a result of this poor connectivity among vehicular nodes is that the effective diameter of the network is small [4].

\section{DSRC Infrastructure}

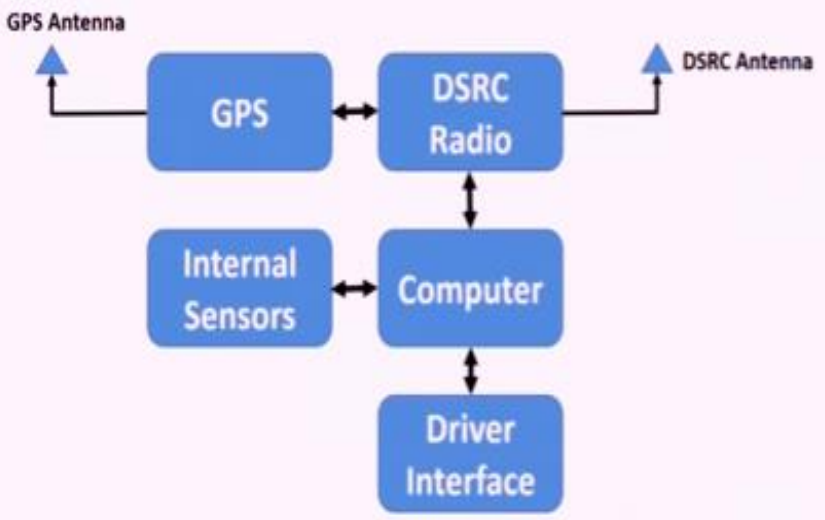

Fig. 2. DSRC infrastucture in VANET 
According to some surveys reports given on VANET environment, probably $80 \%$ commuters use road as primary mode of transportation service. In order to collect toll amount at toll gates the ATTSII (Association of Toll and Traffic Systems Integrators of India) has issued suitable guidelines for ETC [5] (Electronic Toll Collection) service by road safety and traffic management ministry, ministry of surface transport and highways, and electronic toll collection systems. Electronic toll collection means while driver of a vehicle is far away from toll gate, toll operator collects his credit card number or any other required details for transaction and complete that transaction so that driver will go directly without stopping at toll plaza. This feature tremendously saves the time and controls the traffic flow [6]. So, to implement ETC service for VANET, the association proposes a standard based on DSRC microwave technology called as CEN 278 which makes the VANET environment as more reliable, viable, and efficient. CEN DSRC technology was developed to serve multiple applications to facilitate best services in ETC. In this standard, smart tags concept has been introduced based on microwave technology. It saves not only fuel in VANET environment but also reduces waiting time significantly [7].
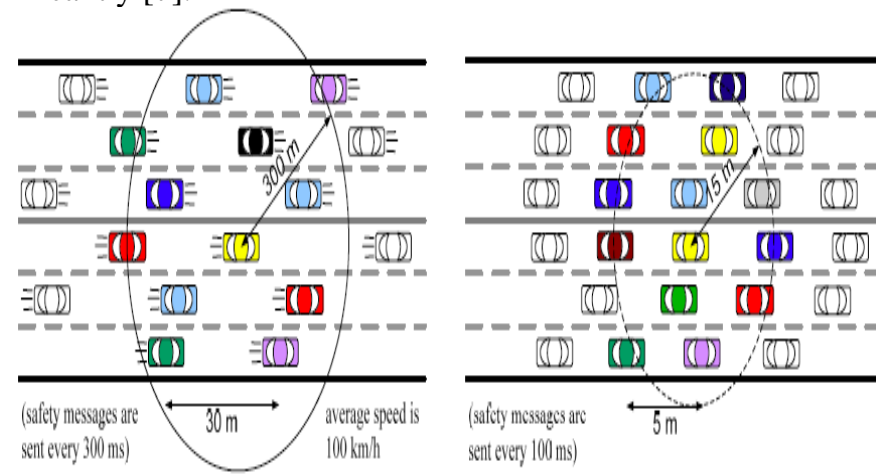

Fig. 3. Moving traffic and congention situations in VANET

Sharing of information in communication means to communicate vehicular nodes freely, monitoring and to enable the users to access better services in all conditions.

\section{A. DSRC Functions, Operating and Benefits}

The rudimentary functions of DSRC in safety messaging and secure communication are:

- Efficient use of spectrum

- $\quad$ Security oriented and prioritized delivery of messages

- Minimization of packet delay as well as authentication

In DSRC standard the main target is sending safety messages among vehicular nodes in VANET with high reliability and full efficiency in order to provide better services to roadside users. DSRC [8] protocol is licensed at $5.9 \mathrm{GHz}$ band with $75 \mathrm{MHz}$ spectrum and divided into seven channels (10 $\mathrm{MHz}$ each) as $1 \mathrm{CCH}$ (control channel), remaining as $6 \mathrm{SCH}$ (Service channels) and a $5 \mathrm{MHz}$ guardband. The $\mathrm{CCH}$ will be used for safety applications and other six SCHs will be used for infotainment or commercial applications. Increasing the $\mathrm{CCH}$ interval will increase the reliability of the safety applications and challenge the coexistence of both safety and non-safety applications on DSRC protocol in VANET environment. In this microwave technology, public transportation vehicular nodes broadcasts using wireless channels and control traffic signals changes that traffic signals according to desired respond in order to give quick respond in emergency and dangerous conditions. In DSRC [9] technology, the appropriate integration of sensors along with GPS positioning system provide tremendous communication capabilities to obtain better services in VANET but raises formidable research challenges. The communication type will be one-to-many, many-to-one, geo significant, and local. Whenever offered traffic is large then; latency, reliability, and channel efficiency will be decreased. In V2V messaging when DSRC involves, all protocols we will be designed are classified into either synchronous or asynchronous messages as:

- $\quad$ SFR (Synchronous Fixed Repetition)

- AFR (Asynchronous Fixed Repetition)

- AFRCS (AFR with Carrier Sensing)

- APR (Asynchronous p-persistence Repetition)

- $\quad$ APRCS(APR with Carrier Sensing)

DSRC was particularly developed for the fulfillment of the requirements of the VANET system environment. It works on physical and MAC layer of IEEE 802.11 standard. It operates on $75 \mathrm{MHz}$ spectrum in $5.9 \mathrm{GHz}$ frequency band at 27 MBPS data rate in US. In Europe, Japan countries it operates on $30 \mathrm{MHz}$ spectrum in $5.8 \mathrm{GHz}$ band. It provides high level data rate transfers of communication with low latency in small zones. The control channel is also used to announce the services that are available. Implementation details are communication range is 300 meters, data rate 6 mbps and broadcast period is $300 \mathrm{~ns}$. It means it serves safety applications. In opposite, service channels serve for nonsafety applications [10].

\section{B. Current working of IEEE standards on DSRC}

Furthermore, IEEE task group is currently working on IEEE 802.11p for both PHY and MAC layers of DSRC. The IEEE 802.11p standard (second generation DSRC) rectifies all problems which are existed in first generation DSRC [9]
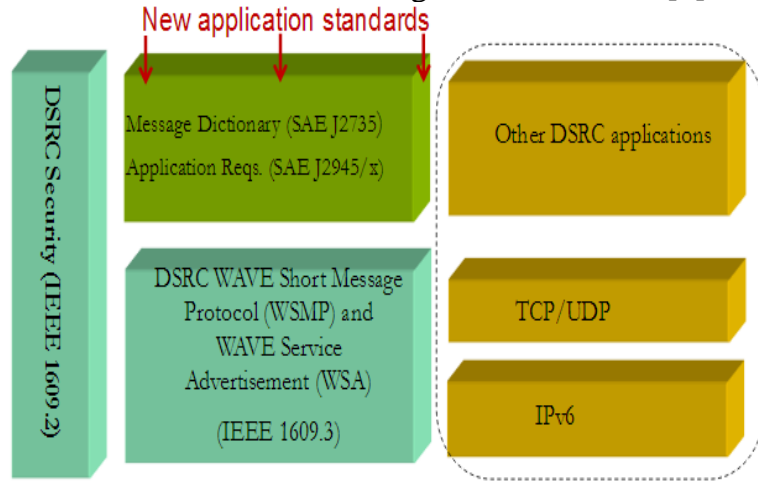

DSRC Multi-Channel MAAC (IEEE 1609.4)

Next Gen. V2X starting at IEEE

Fig. 4. DSRC standards for VANET system

This paper is organized into IV sections. First section tells about introduction, second section discusses on some related work, third section explains about methods used, and section 
four describes conclusion. At last references are given which are used throughout the paper.

\section{LITERATURE SURVEY}

A number of unique applications are developed for DSRC technology. Many analytical models have been proposed to study the DSRC using IEEE 802.11 and EDCA (Enhanced Distributed Channel Access) for broadcast messages among vehicular nodes. Torrent - Moreno et al. [11] and Vaneenennaam et al. [12] introduced an algorithm to control the load of periodic messages. The channel delay of the DSRC has been analyzed by K. Bilstrup [13] and compared with a self organizing TDMA (Time Division Multiple Access) which has been proven more suitable for VANET real time applications. Wang and Hassan [14] proposed a framework for sharing the DSRC between vehicular safety and non-safety applications. By assuming uniformdistribution of vehicular nodes on the road show that their simulation results of non safety applications are not compromised, especially in high density networks. S. Eichler [15] analyzed the DSRC based on average delay in distributing a safety message to destination in VANET system. Frachcchia and M. Meo [16] analyzed a model for delivering safety messages using IVC. They assume perfect channel for deliver of message which was derived from IVC based on vehicular node mobility. Fallah et al. [17] analyzed the effect of different sets of data rates and communication ranges on the performance of the DSRC safety applications. Here, they introduced a power control algorithm based on the average channel occupancy which has been used only changed communication range. In this algorithm if channel occupancy increases they decrease the communication range and vice-versa to maintain the acceptable channel capacity. Venkatamangarao Nampally and Dr. Raghavender Sharma have achieved safety transmission of a message by using NTBS protocol in VANET thus to increase communication flow in VANET [18].

\section{METHODOLOGY}

\section{A. DSRC Channel Allocation}

The FCC allocated 5.9 GHz band as new DSRC.

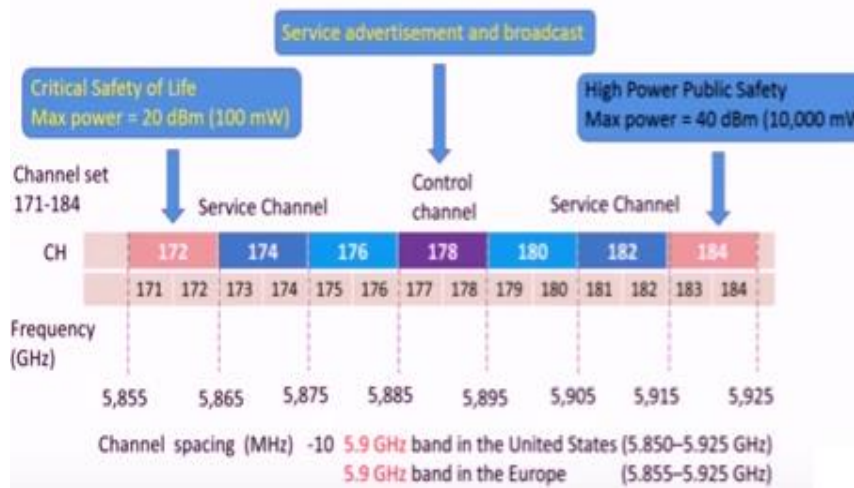

Fig. 5. DSRC channels for VANET system

In order to provide best service facilities in VANET system environment, first generation DSRC divided into two channels. Whereas second generation DSRC divided into seven channels each at $10 \mathrm{MHz}$. In these channels one is control channel and remaining six are service channels. 915
$\mathrm{MHz}$ DSRC supports a data rate of $0.5 \mathrm{Mb} / \mathrm{s}$ but $5.9 \mathrm{GHz}$ DSRC supports 6 to $27 \mathrm{Mb} / \mathrm{s}$. Thus two channels, the data can reach at maximum $54 \mathrm{Mb} / \mathrm{s}$ when two channels are combined to form one $\mathrm{MHz}$ channel..It is composed of seven channels as shown above figure each channel at $10 \mathrm{MHz}(1$ $\mathrm{CCH}$ and $6 \mathrm{SCH})$. The modulation scheme used by DSRC is OFDM (Orthogonal Frequency Division Multiplexing. Above figure provides channel layout of DSRC. The list of channel applications supported by that channel is:

- Channel 172 is reserved for medium power safety applications.

- Channel 174 is reserved for medium power safety applications shared by all.

- Channel 175 is combination of both 174 and 176 channels.

- Channel 176 is reserved for medium power applications shared by all.

- Channel 178 is the control channel which supports all power levels, safety application broadcasts, service announcements and vehicle-to-vehicle broadcasts messages.

- Channel 180 is reserved for low power configurations and provides little interference when vehicles are separated by $50 \mathrm{ft}$ or more.

- Channel 181 is combination of channels 180 and 182.

- Channel 182 is reserved for low power configurations and provides little interference when vehicular units are separated by $50 \mathrm{ft}$ or more.

- Channel 184 is reserved for a high power service channel that is used to co-ordinate intersection applications.

The current wireless technology is only able to listen to only one transceiver. To overcome this problem, to equip an OBU or a RSU with multiple transceivers allow them access to multiple channels simultaneously. The drawback of having multiple radios is it increases the cost and complexity [19].

\section{B. First and Second Generation DSRCs}

The first generation of DSRC operates at $915 \mathrm{MHz}$ at transmission rate $0.5 \mathrm{Mb} / \mathrm{s}$. it is very scalable and efficient offers low cost facilities to its users. It has only one or two channels in order to provide service. One of examples for first generation DSRC application is EZpass which is used for electronic toll collection. But this project achieved limited success rate and is primarily used for electronic toll collection for commercial vehicles in VANET. In first generation bandwidth of DSRC protocol is not sufficient in all situations. So FCC allocated additional $75 \mathrm{MHz}$ bandwidth in $5.9 \mathrm{GHz}$ band. This new tremendous amendment is called second generation DSRC. The second generation DSRC system started in 1997 and it is operated at $75 \mathrm{MHz}$ of bandwidth in the $5.9 \mathrm{GHz}$ band allocated by FCC (Federal Communication Commission). The primary goal of this second generation DSRC is to provide up-to-date information regarding surrounding environment to users in VANET and decrease traffic congestions, accidents on highway road. In this DSRC, high dynamicity is possible due to high achievable vehicle mobility by the GPS enabled vehicles that are provided with on-board-units connected with each other on VSC (Vehicle Safety Communication) technology [20]. Many opportunities 
have become possible by transferring safety messages via communication capabilities of integrated GPS and on-board sensors when IVC has been taken into consideration. In IVC each vehicular node equipped with communication receiving and sending capability devices with appropriate sensors, GPS to collect information about their speed, acceleration, position and direction to be broadcasted to all vehicular nodes with their radio signals coverage. Generally, where communication zones which have relatively small in geographic area, they require high data transfer rate. In these areas, first generation DSRC is not sufficient to make vehicular nodes proper communication in VANET system. So, FCC allocated $5.9 \mathrm{GHz}$ band at $75 \mathrm{MHz}$ spectrum. Second generation DSRC provides data transfer rate at 6 $\mathrm{Mb} / \mathrm{s}$ to $27 \mathrm{Mb} / \mathrm{s}$ per channel [21]. So merging of four channels provide approximately $100 \mathrm{Mb} / \mathrm{s}$ data transfer rate. According a particular zone requirement, we use channels as data transfer rate requirement. SAE J2735 is a standard in DSRC for defining messages in VANET. It defines approximately 15 messages. But the main transmitted message types in DSRC (SAE J2735) are [22]:

- $\quad$ TIM (Traveler Information Message)

- SPaT (Signal Phase and Timing)

- MAP (Map Data Message)

- PVD (Probe Vehicle Data message)

- $\quad$ BSM (Basic Safety Message)

The $5.9 \mathrm{GHz}$ DSRC overcomes many weaknesses of 915 MHZ DSRC. The following table describes important features comparison between first and second generation DSRCs.

TABLE I. COMPARISON BETWEEN DSRC TECHNOLOGIES

\begin{tabular}{|c|c|c|}
\hline & $\mathbf{9 0 2 - 9 2 8} \mathbf{M H Z}$ Band & $\mathbf{5 8 5 0 - 5 9 2 5} \mathbf{M H z}$ Band \\
\hline Spectrum & $12 \mathrm{MHz}$ & $75 \mathrm{MHz}$ \\
\hline Coverage & $\begin{array}{c}\text { One communication } \\
\text { zone }\end{array}$ & $\begin{array}{c}\text { Overlapping } \\
\text { communication zones }\end{array}$ \\
\hline Data transfer rate & $0.5 \mathrm{Mb} / \mathrm{s}$ & $\begin{array}{c}6 \text { to } 27 \mathrm{Mb} / \mathrm{s} \text { per } \\
\text { channel }\end{array}$ \\
\hline Channels & 1 or two & 7 \\
\hline Downlink power & Less than $40 \mathrm{dBm}$ & Less than $33 \mathrm{dBm}$ \\
\hline Uplink power & Less than $6 \mathrm{dBm}$ & Less than $33 \mathrm{dBm}$ \\
\hline $\begin{array}{c}\text { Interference } \\
\text { potential }\end{array}$ & High & Low \\
\hline Maximum range & $300 \mathrm{ft}$ & $1000 \mathrm{ft}$ \\
\hline $\begin{array}{c}\text { Minimum } \\
\text { separation }\end{array}$ & $1500 \mathrm{ft}$ & $50 \mathrm{ft}$ \\
\hline
\end{tabular}

\section{DSRC characteristics and Category of applications}

The essential key benefits of DSRC microwave technology are: two powerful technologies availability (IEEE 802.11a and IEEE 802.11p), seven licensed channels availability, high data transfer rate, low latency communication $(<<50 \mathrm{~ms})$, and low power message reception. A large array of applications are developed and being developed for Dedicated Short Range Communication. These applications of DSRC [23] are categorized into four classes. These are:
- $\quad$ Lane Change Warning

- Wrong Way Driver Warning

- Cooperative Adaptive Cruise Control

- Emergency electronic Brake Lights

- Do Not Pass Warning

- Intersection Collision Warning

- Vehicle-to-infrastructure safety

- Right Turn Assist

- Left Turn Assist

- Pedestrian Signal Assist

- Red Light Running

- Rail Crossing

- Transit Signal Priority

- Emergency vehicle Preempt

- $\quad$ CVO (Commercial Vehicle Operation)

- Driver Log

- Border Crossing

- Fleet management

- Inventory and Container Management

- Wireless Inspection

- Control Loss Warning

- Vehicle Diagnostics

- Weigh in Motion Stations

- $\quad$ Private applications

- Parking Management

- Service Record

- Vehicle Diagnostics

- Rental Car Transactions

- Fuel/Drive-thru Management

- Access Control

- Advanced Traveler Information Sys.

- Probe/Data/ Traffic Information

Besides these four the DSRC applications further categorized as safety and non-safety applications. The function of the public safety application is the improvement of the overall safety transportation infrastructure. The public safety applications allow protection to the safety of life, and health of users. These public safety services are provided by either governmental or non-governmental agencies under the control of authorized governmental body. As a survey DSRC provides approximately 34 possible safety services to users through DSRC. And the function of the non-safety applications increase the comfort of the user by adding value added services. It is clear that public safety applications are always given priority than non-safety applications. The message of DSRC is either event or periodic. Event-driven message means it is delivered only some event occurred while periodic messages transmitted at a special interval. In order to gain widespread adoption and to provide interoperability feature DSRC supports number of different protocols. On the basis of evaluated criteria, the VSC project determined the safety applications as the highest priority based applications to implement in VANET system environment to save the lives of its users.

- Vehicle-to-Vehicle safety

- Forward Collision Avoidance

- Blind Spot Warning 


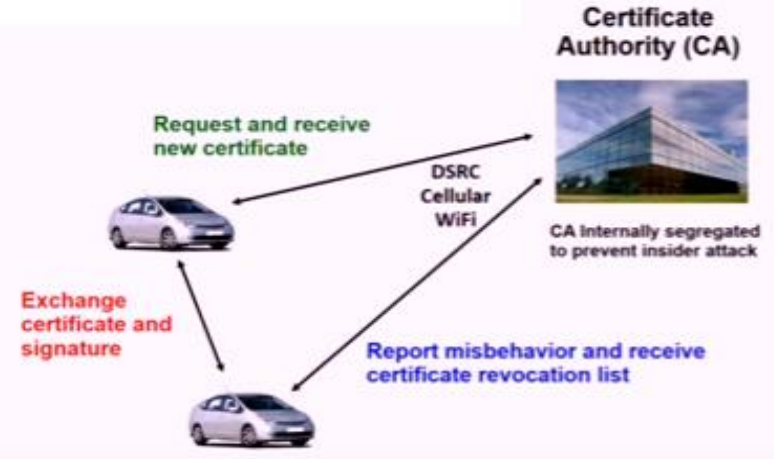

Fig. 6. DSRC certificate infrastucture

\section{Technologies Used for DSRC}

in order to implement wireless technology, it is a very important a requirement is it must have its latency $100 \mathrm{~m}$ or less than $100 \mathrm{~m}$. it offers high throughput and have communication range $100 \mathrm{~m}$ to $1000 \mathrm{~m}$. we can increase the communication range by using better protocol. The wireless technology should support both one way and two way communication. And it must support combination of both to establish a dialog among them.

\begin{tabular}{|l|l|l|l|l|}
\hline & DSRC/WAVE & Wi-fi & Cellular & Mobile WiMAXS \\
\hline Data rate & $3.27 \mathrm{Mbps}$ & $6.54 \mathrm{Mbps}$ & $<2 \mathrm{Mbps}$ & $1.32 \mathrm{Mbps}$ \\
\hline Mobility & $>60 \mathrm{mph}$ & $<5 \mathrm{mph}$ & $>60 \mathrm{mph}$ & $>60 \mathrm{mph}$ \\
\hline $\begin{array}{l}\text { Nominal } \\
\text { Bandwidth }\end{array}$ & $10 \mathrm{MHz}$ & $20 \mathrm{MHz}$ & $<3 \mathrm{MHz}$ & $<10 \mathrm{MHz}$ \\
\hline Operating Band & $\begin{array}{l}5.86-5.92 \mathrm{GHz} \\
(\text { ITS-RS) }\end{array}$ & $\begin{array}{l}2.4 \mathrm{GHz}, 5.2 \mathrm{GHz} \\
\text { (ISM) }\end{array}$ & $800 \mathrm{MHz}, 1.9 \mathrm{GHz}$ & $2.5 \mathrm{GHz}$ \\
\hline IEEE std. & $\begin{array}{l}802.11 \mathrm{p} \\
\text { (WAVE) }\end{array}$ & $802.11 \mathrm{a}$ & $\mathrm{N} / \mathrm{A}$ & $802.16 \mathrm{e}$ \\
\hline
\end{tabular}

Fig. 7. DSRC complementary technologies

In the end a modified version of 802.11a was chosen as primary means of communication of DSRC. Number of other evaluated wireless technologies was unacceptable for various reasons.

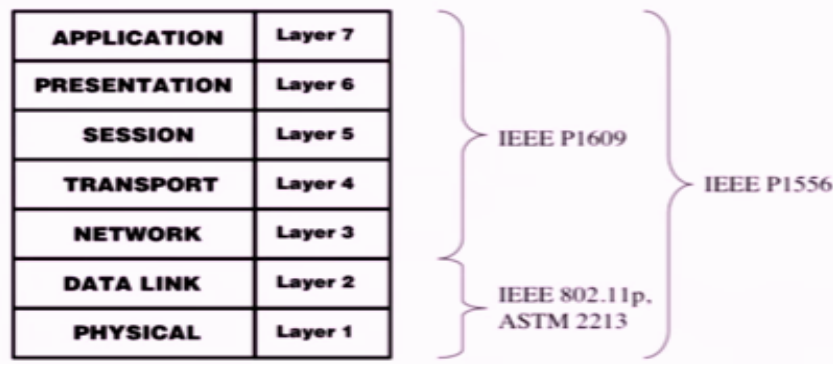

Fig. 8. DSRC protocol stack

Reason behind huge use of wireless technology in VANET is its cost must be low than both cellular and satellite technologies. And also wireless technology is based on ad hoc networks and infrastructure of wireless costs us very cheap than cellular and satellite technologies. Below table compares wireless with cellular and satellite technologies.

TABLE II. DSRC COMPLEMENTARY TECHNOLOGIES

\begin{tabular}{|c|c|c|c|}
\hline & DSRC & Satellite & Cellular \\
\hline Range & $100 \mathrm{~m}-1000 \mathrm{~m}$ & $10^{3}$ kilometers & Kilometers \\
\hline Cost & Very low & Very Expensive & Expensive \\
\hline Latency & $200 \mu \mathrm{s}$ & 10 to $60 \mathrm{~s}$ & 1.5 to $3.5 \mathrm{~s}$ \\
\hline
\end{tabular}

A major problem in DSRC is dissemination of new maps or updated route of map of a new city which is never visited before or when a road is altered. In order to overcome this problem DSRC uses many technologies than wireless. A tremendous technology GPS provides great solution to the problem of determining vehicle location. But GPS will not work properly in all situations because it requires a clear path to satellite. It is not possible in all times. Sensor technology used for provides additional input to the VANET system environment. In other words, vehicular nodes and all infrastructure of VANET to give and obtain better communication services use the sensors which monitor the local conditions in appropriate places. In future, it is better if we use radar technology. A radar device is added to RSU so that it is able to detect vehicles lacking a DSRC transceiver. Then RSU relay location information to all non-DSRC equipped vehicles along with DSRC equipped vehicles. In VANET system, each RSU requires a license in order to maintain the integrity in VANET. An RSU can communicate with another RSU using wired infrastructure to make the communication more reliable and working condition of RSU is more efficient. The IEEE 802.11 standard uses the concept of slot time. And this family of protocols uses CSMA/CA (Carrier Sense Multiple Access with Collision Avoidance) as acknowledgement to restrict the number of collisions and to reliably transmit packets. NHTSA (National Highway Traffic Safety Administration) of US defined vehicle levels as:

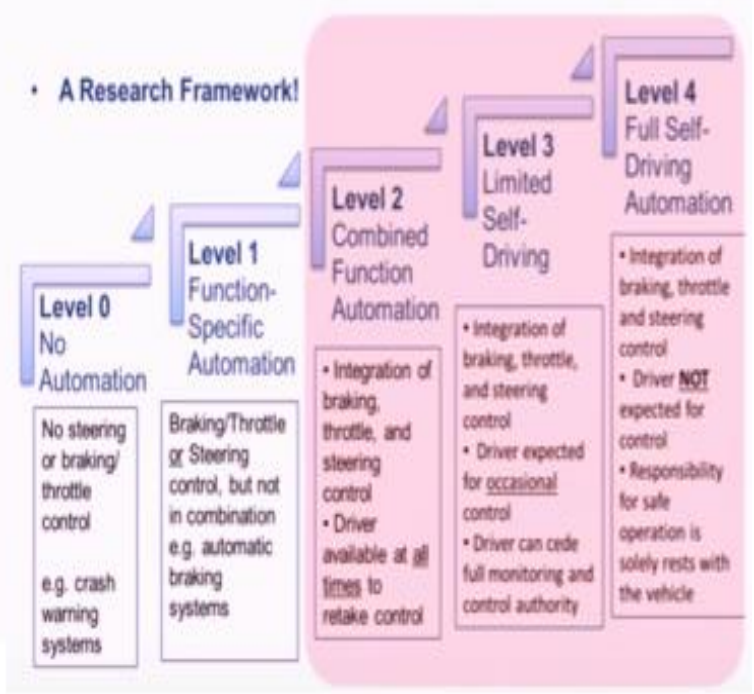

Fig. 9. NHTSA Automated vehicle levels

\section{E. MAC protocols used in VANET}

Media Access Protocols such as TDMA, FDMA, and CDMA are difficult to implement in VANET system. To use of these protocols channels, codes, or time-slots needs to be 
implemented dynamically. Theoretically, the objective of the MAC layer is to attribute the access to the shared medium. The MAC layer helps users in controlling the collisions as it prevents the nodes within the transmission range of each other from transmitting at same time. The IEEE 802.11 standard defines two MAC protocols DCF (Distributed Coordination Function) and PCF (Point Coordination Function). The DCF protocol is asynchronous contention based protocol whereas PCF is contention free protocol. Contention based means all nodes that have data to send contend for access to the engaged channel. On other hand, contention protocols such as PCF enable the use of real-time services. Contention free protocols provide access to the medium by scheduling when a node can transmit. But PCF relies on the central node to support the real-time delivery of packets. So, for this reason the majority of communication that takes place in DSRC uses the DCF. Moreover, the DCF achieves collision avoidance with a random back-off procedure. When a node begins a transmission, it randomly selects the number of slots it must wait before transmitting. The process is called as back-off procedure. Generally time slot will depend on PHY layer characteristics of the IEEE 802.11 protocol and this value is for IEEE 802.11a standard is $9 \mu \mathrm{s}$. if two nodes want to transmit the packet at same time then they select different values for their back-off timers thus they avoid collision.
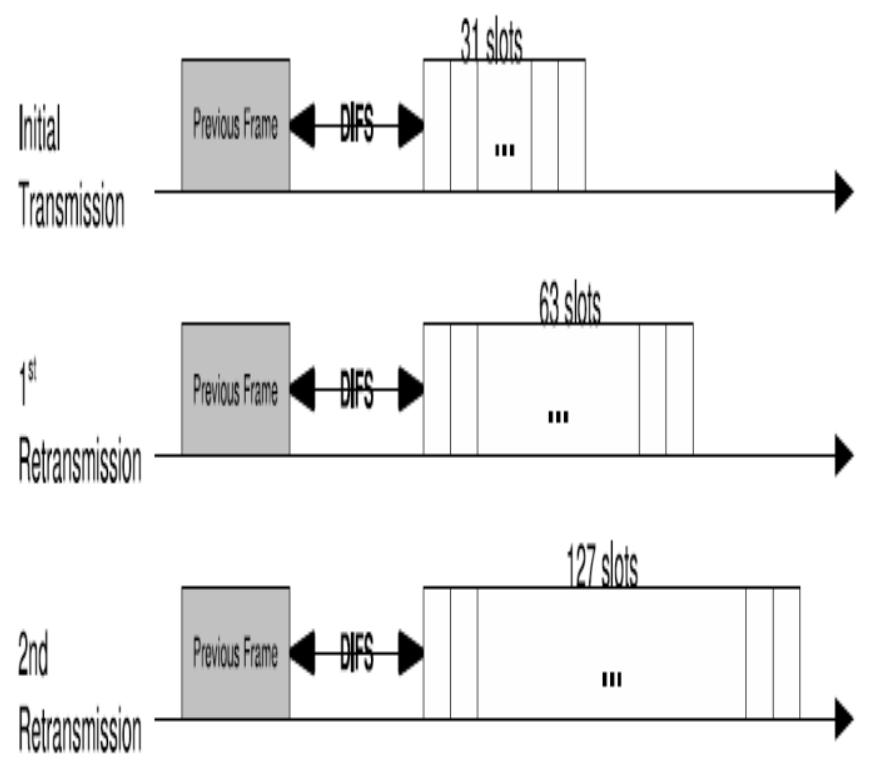

Fig. 10. Back-off procedure in VANET IEEE 802.11 standard

\section{F. DSRC importantstandards}

The VSC3 consortium of automotive manufacturers has begun a project for testing of scalability and interoperability of DSRC technology. The IEEE 802.11p standard is the amendment of IEEE 802.11 standard (WAVE).

TABLE III. DSRC STANDARDS AND DISCRIPTION

\begin{tabular}{|c|c|}
\hline DSRC Standards & Description \\
\hline SAE J2735 & Messaging, Message set Dictionary \\
\hline SAE J2945 & Safety Awareness Applications \\
\hline IEEE 1609.1 & Remote management \\
\hline IEEE 1609.2 & For Security \\
\hline
\end{tabular}

\begin{tabular}{|c|c|}
\hline IEEE 1609.3 & Network services including WSMP \\
\hline IEEE 1609.4 & $\begin{array}{c}\text { Multi-Channel operation, channel } \\
\text { switching }\end{array}$ \\
\hline IEEE 1609.11 & For ETC \\
\hline IEEE 1609.12 & PSID allocations \\
\hline IEEE $802.11 \mathrm{p}$ & Services to ITS \\
\hline
\end{tabular}

\section{CONCLUSION}

The DSRC technology can be helpful in future generation transportation systems without any doubt. The main function of DSRC microwave technology is highly dependent on cooperative standards for interoperability. In this paper, we have discussed DSRC in possible dimensions. The DSRC technology will facilitate connected autonomous vehicle environment in VANET with safety enable feature. It means in this state, the vehicle provides all advanced and modern technology features autonomously. DSRC standard is tremendous powerful protocol for VANET communication. We also discussed MAC protocols used for providing best service properly broadcast a safety message among vehicles and infrastructure. There are many other protocols to address the various problems in VANET system communication. The standards of DSRC are being updated day-to-day. Currently, we are controlling $70 \%-80 \%$ accidents using DSRC technology throughout the globe. DSRC has unique characteristics to enable intelligent transportation system safely. Currently, groups such as VSCC (Vehicle Safety Communication Consortium) are working to invent new protocols for DSRC. And another group CAMP (Crash Avoidance Metrics Partnership) is an automobile manufacturer including GM and ford, which is working on the realization of the collision avoidance components for DSRC. The CAMP IVI (CAMP Intelligent Vehicle Initiative) is a research program that brings together a number of automobile manufacturers and supplies the work cooperatively with US DOT (US Department of Transportation). In this connection, there are some concerns in widespread deployment but progress in technology. The technologies invented now, may not implement in this generation because VANET provides unlimited opportunities but may implement in future. And future VANET system, definitely offers tremendous opportunities and best services to its users through tremendous protocols to increase the transportation facility by reducing traffic fatalities and vehicles collisions. US DOT wants $100 \%$ free accident zone by 2050 using this tremendous technology involving new powerful protocols.

\section{REFERENCES}

[1] Venkatamangarao Nampally, Dr. M. Raghavender Sharma, "Information sharing standards in communication for VANET", International Journal of Scientific Research in Computer Science Applications and Management Studies (IJSRCSAMS), Vol. 7, Issue. 4, 2018, ISSN: 2319-1953, GIF: 0.6.

[2] Federal Communications commision. Amendment of the commision's rules regarding dedicated short range communication service in the 5.850-5.925 GHz band, FCC 02-302.Tech.Rep., FCC, Nov.2002.

[3] NHTSA. Intelligent Transportation Systems, 2006.

[4] H. Moustafa, and Y. Zhang, "vehicular network techniques, standards, and applications, CRC press, 2009.

[5] https://www.itsstandards.eu/efc online link 
[6] C. Campolo, A. Molinaro, A. Vinel, and Y. Zhang, "Modeling prioritized broadcasting in multichannel vehicular networks, IEEE Tran. Veh. Technol., Vol.62, No. 2, pp. 687-701, Feb.2012.

[7] https://www.itsstandards.eu/tc278 online link

[8] Dedicated Short Range Communications (DSRC), Available:http://grouper.ieee.org/groups/scc32/dsrc/index.html

[9] J. Kenney, "DSRC standards in United States", Proc.IEEE, Vol. 99 No. 7,pp.1162-1182, July 2011.

[10] https://www.itsstandards.eu/dsrc online link

[11] M. Torrent-Moreno, J. Mittag, P. Santi, and H. Hartenstein, "Vehicle-to-vehicle communication: Fair transmit power control for safety critical information", IEEE Trans. on Veh. Technol., Vol.58, No.7, pp. 3684-3703, sep.2009.

[12] E. M. Vaneenennaam, W. kleinwolterink, G. Karageannis, and G. J. Heijenk, "Exploring the solution space of beaconing in VANETs", in the proceedings of the $1^{\text {st }}$ IEEE VNC, Tokyo, Japan, pp.1-8, 2009.

[13] K. Bilstrup, E. Uhlemann, E. G. Strom, and U. Bilstrup, "Evalution of the IEEE 802.11p MAC method for vehicle-to-vehicle communication", in the proceedings of the IEEE $68^{\text {th }}$ Veh. Technol. Conf., pp.1-5, 2008.

[14] Z. Wang and M. Hassan, "How much of DSRC is available for nonsafety use?", in the proceedings of $5^{\text {th }}$ ACM. Int. Workshop. Veh. Inter. NETw., pp.23-29, 2009.

[15] S. Eichler, "Performance evalution of the IEEE 802.11p WAVE communication standard", in the proceedings of the IEEE Veh. Techlo. Conf., pp.2199-2203, 2007.
[16] Fracchia and M. Meo, "Analysis and design of warning delivery service in Inter vehicular Networks", IEEE Trans. Mobile Compute. Vol.7, No.7, pp.832-845, Jul. 2008

[17] P. Fallah, H. Ching-Ling, R. Sengupta, and H. Krishnan, "Analisis of information dissemination in vehicular ad-hoc networks with application to co-operative vehicle safety systems", IEEE Tran. Veh. Technol., Vol.60, no.1, pp.233-247, Jan.2011.

[18] Venkatamangarao Nampally, Dr. M. Raghavender Sharma "Increasing Information Shareability by Using NTBS Clustering Approach for VANET', IPASJ International Journal of Computer Science ( IIJCS), Vol.5, Issue.10, pp.1-17, 2017.

[19] M. I. Hassan, H. L. Vu, and T. Sakurai, "Performance analysis of the IEEE 802.11 MAC protocol for DSRC safety applications", IEEE Trans. Veh. Technol., Vol. 60, No. 8, pp.3882-3896, Oct. 2011.

[20] http://nathanbalon.net/projects/cis695/vanet_chapter.pdf

[21] Khalid Abdel Hafeez, and Bobby Ma, "Performance and enhancement of the DSRC for VANET's safety applications", IEEE transactions on Vehicular Technology, Vol.62, No.7, September 2013.

[22] G. Badawy, J. Misic, T. Todd, and D. Zhao, "Performance modeling of safety message delivery in vehicular ad hoc networks", in the proceedings of the IEEE $6^{\text {th }}$ Int. Conf. WiMob., pp.188-195, Oct. 2010.

[23] Armstrong, L. Dedicated short Range Communications Project. 